\title{
Flora da Bahia: Catasetum (Orchidaceae)
}

\author{
Cláudia Araújo Bastos* \& Cássio van den Berg
}

Universidade Estadual de Feira de Santana, Departamento de Ciências Biológicas, Av. Transnordestina, s/n, Novo Horizonte, 44036-900, Feira de Santana, Bahia, Brasil.

\begin{abstract}
Resumo - É apresentado o levantamento florístico de Catasetum (Orchidaceae) do estado da Bahia, Brasil. Foram reconhecidas seis espécies: C. blackii, C. hookeri, C. luridum, C. macrocarpum, C. purum e C. roseo-album. O tratamento inclui descrições dos táxons, além de chave de identificação, ilustrações e comentários para as espécies.

Palavras-chave adicionais: Brasil, florística, orquídea, taxonomia.
\end{abstract}

\begin{abstract}
Flora of Bahia: Catasetum (Orchidaceae)) - The floristic survey of Catasetum (Orchidaceae) from the state of Bahia, Brazil, is presented. Six species were recognized: C. blackii, C. hookeri, C. luridum, C. macrocarpum, C. purum, and $C$. roseo-album. The treatment includes description of taxa, as well as key for identification, illustrations, and commentaries for species.
\end{abstract}

Addition key words: Brazil, floristics, orchid, taxonomy.

\section{Orchidaceae}

Ervas terrestres, epífitas, ou trepadeiras. Pseudobulbos presentes ou caule curto. Folhas solitárias a numerosas. Inflorescência terminal ou lateral, flor solitária a panícula densa, pedúnculo pendente ou ereto; brácteas escapais presentes ou não. Flores ressupinadas ou não, zigomorfas; sépalas 3 ; pétalas 3 , sendo a mediana modificada em labelo; labelo simples a trilobado, provido ou não de calosidades ou adornos; coluna com estames e pistilo fundidos, parte do estigma às vezes modificada em rostelo; polinário com 2, 4, 6 ou 8 polínias; ovário ínfero. Fruto cápsula. Sementes numerosas, minúsculas.

De distribuição cosmopolita, Orchidaceae é encontrada principalmente nas regiões tropicais. A família inclui aproximadamente 850 gêneros e ca. 20.000 espécies (Dressler 1993; Pridgeon et al. 1999), sendo que no Brasil ocorrem ca. 200 gêneros e 2.500 espécies (Pabst \& Dungs 1975, 1977; Barros et al. 2010). Para a Bahia, Pabst \& Dungs $(1975,1977)$ citaram ca. 150 espécies, ocorrendo nos mais variados ambientes, enquanto Barros et al. (2010) listaram ca. 400 espécies para o estado.

\section{Catasetum Rich. ex Kunth}

Erva epífita ou terrestre. Pseudobulbos fusiformes, eretos, homoblásticos, cobertos por numerosas bainhas paleáceas. Folhas plicadas, planas, cartáceas, concolores, lanceoladas a ovais, atenuadas na base, agudas no ápice. Inflorescência lateral axilar às bainhas do pseudobulbo, racemosa, multiflora; pedúnculo pendente, cilíndrico; brácteas escapais elípticas. Flor estaminada ressupinada ou não, em geral grande, exuberante, aromática; brácteas florais lanceoladas; sépalas e pétalas membranosas, lanceoladas a ovais; labelo geralmente côncavo, às vezes explanado, trilobado ou lobos indefinidos, lobos lisos ou

"Autora para correspondência: caubionet@yahoo.com.br

Editor responsável: Pedro Fiaschi

Submetido em: 25 nov. 2010; publicação eletrônica: 11 jun. 2012 com fímbrias, serras ou calosidades; coluna alongada, ereta ou arqueada; antenas 2; antera com polinário formado por 2 polínias, caudículos, estipe e viscídio; flor pistilada não ressupinada, menos atrativa; sépalas e pétalas membranosas a carnosas, oblongas a lanceoladas; labelo côncavo, calceolado a campanulado, sem lobos definidos, sem fímbrias, serras ou calosidades; coluna curta; antenas ausentes ou rudimentares; antera atrofiada, caduca.

O gênero neotropical Catasetum inclui 130 espécies, sendo o Brasil a principal região de ocorrência, com ca. de 100 espécies listadas (Barros et al. 2010). Para a Bahia, são citadas de seis a 12 espécies (Pabst \& Dungs 1975; Holst 1999; Barros et al. 2010), sendo que aqui foram reconhecidas seis. Espécies faltantes em relação a outros levantamentos incluem Catasetum barbatum (Lindl.) Lindl., C. gnomus Linden \& Rchb.f. e C. mattosianum Bicalho, que não foram registradas nos herbários estudados.

O gênero apresenta notável dimorfismo floral e as flores pistiladas são sempre muito semelhantes entre as espécies, enquanto que as flores estaminadas, com estruturas em forma de antenas na face ventral da coluna, fundamenta a taxonomia do gênero (Bicalho 1960; Toscano de Brito \& Cribb 2005).

\section{Chave de identificação}

1. Labelo bilobado; pétalas com máculas castanhas........... 4. C. macrocarpum

1'. Labelo trilobado; pétalas sem máculas.

2. Sépalas e pétalas patentes; labelo membranoso, lobos laterais revolutos ou eretos, lobos laterais e mediano de margem fimbriada.

3. Epífita; flor ressupinada; labelo liguliforme, lobo mediano com calosidade linear na face adaxial; antenas $0,7-0,8 \mathrm{~cm}$ compr....................1. C. blackii

3'. Terrestre; flor não ressupinada; labelo cuculado, lobo mediano sem calosidade; antenas inconspícuas 6. C. roseo-album

$2 '$. Sépalas e pétalas eretas; labelo carnoso, lobos laterais involutos, de margem serreada ou denticulada e lobo mediano com margem inteira.

Sitientibus série Ciências Biológicas 12(1): 83-89. 2012. 
4. Labelo com abertura pandurada, lobo mediano involuto 5. C. purum

4'.Labelo com abertura oval, lobo mediano levemente dobrado para frente.

5.Flor ressupinada; lobo mediano arredondado 3. C. luridum

5'. Flor não ressupinada; lobo mediano oval 2. C. hookeri

1. Catasetum blackii Pabst, Anais 14 Congr. Soc. Bot. Brasil: 21. 1964.

Figuras $1 \mathrm{e} 2 \mathrm{~A}-\mathrm{D}$.

Erva epífita. Pseudobulbo ca. $10,5 \times 1,5 \mathrm{~cm}, 5$ entrenós. Folhas 27,6-36,5 × 2,3-6,2 cm, lanceoladas. Inflorescência ca. 18 flores; pedúnculo 48,4-61,2 $\times$ $0,5-0,6 \mathrm{~cm}$; brácteas escapais $6,0,8-1,1 \times 0,3-0,6 \mathrm{~cm}$. Flor estaminada ressupinada; brácteas florais $9-18$, ca. $0,8 \times 0,3$ $\mathrm{cm}$; sépala dorsal 3-3,4 $\times 0,8-1,1 \mathrm{~cm}$, patente; sépalas laterais $2,8-3,4 \times 0,7-0,9 \mathrm{~cm}$, patentes; pétalas $2,6-3,6 \times$ ca. $0,6 \mathrm{~cm}$, sem máculas, patentes; labelo $1,8-2,1 \times 1-1,7 \mathrm{~cm}$, livre, membranoso, trilobado, semiexplanado, sem nervuras, lobos laterais ca. $0,7 \times 1 \mathrm{~cm}$, semiarredondados, revolutos, margem fimbriada; lobo mediano 1,1-1,4 $\times$ $0,6-1,3 \mathrm{~cm}$, plano, liguliforme, calosidade linear na face adaxial, margem fimbriada; coluna $1,2-1,7 \times$ ca. $0,3 \mathrm{~cm}$, livre; polinário ca. $0,8 \times 0,3 \mathrm{~cm}$; antenas $0,7-0,8 \mathrm{~cm}$ compr.; flor pistilada não vista.

Foi considerada por Holst (1999) uma espécie rara da região do entorno do rio São Francisco, citada apenas para o sudoeste da Bahia e nordeste de Minas Gerais (Barros et al. 2010), mas ocorre também na Chapada Diamantina, conforme Toscano de Brito \& Cribb (2005), e o material aqui relacionado. F6, G5: caatinga. Floresce de novembro a março.

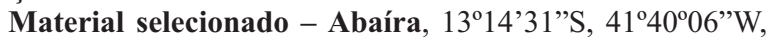
mar. 2004 (fl. O'), A.L.V. Toscano 1824 (RB); Caetité, 28 nov. 1992 (fl. O'), M.L. Guedes et al. s.n. (ALCB 024306).

Espécie epífita comumente encontrada sobre licuri [Syagrus coronata (Mart.) Becc.] e de fácil identificação. Caracteriza-se pelas flores estaminadas ressupinadas, labelo trilobado, raso, com fímbrias na borda, e lobo mediano liguliforme, com uma calosidade linear na face adaxial.

2. Catasetum hookeri Lindl., Bot. Reg. 10: sub. t. 840 . 1821.

Figuras $1 \mathrm{e} 2 \mathrm{E}-\mathrm{H}$.

Erva epífita. Pseudobulbo 7,5-17 × 0,8-2,2 cm, 2-4 entrenós. Folhas 15,9-32 × 2,8-6,2 cm, lanceoladas. Inflorescência 3-17 flores; pedúnculo 27,5-56,3 × 0,3-0,6 $\mathrm{cm}$; brácteas escapais 5 ou $6,0,8-1,5 \times 0,4-0,7 \mathrm{~cm}$. Flor estaminada não ressupinada; brácteas florais 6-24, 0,7-1 $\times$ $0,3-0,4 \mathrm{~cm}$; sépala dorsal $1,4-2,9 \times 0,5-1,1 \mathrm{~cm}$, ereta; sépalas laterais $1,6-2,7 \times 0,7-1,6 \mathrm{~cm}$, eretas; pétalas 1,5-2,3 × 0,6-1,8 cm, sem máculas, eretas; labelo 1,3-1,7× 0,5-2,1 cm, livre, carnoso, trilobado, calceolado, abertura oval entre os lobos, sem nervuras; lobos laterais $0,3-1,1 \times$ $0,4-0,9 \mathrm{~cm}$, arredondados, involutos, margem posteriormente serreada, lobo mediano $0,4-1 \times 0,3-0,8 \mathrm{~cm}$, levemente dobrado para frente, oval, sem calosidade, margem inteira; coluna $1,1-1,3 \times 0,3-0,4 \mathrm{~cm}$, envolvida parcialmente pelos lobos laterais; polinário $0,4-1 \times$ ca. 0,3 $\mathrm{cm}$; antenas $1,5-1,7 \mathrm{~cm}$ compr.; flor pistilada não ressupinada; sépalas suculentas, a dorsal $0,6-1 \times$ ca. $0,5 \mathrm{~cm}$, as laterais $07-1,3 \times 0,6-0,8 \mathrm{~cm}$; pétalas $0,7-1,3 \times 0,4-0,8$ $\mathrm{cm}$, suculentas; labelo $0,7-1,8 \times 0,7-1,5 \mathrm{~cm}$, calceolado, suculento; coluna ca. $0,5 \times 0,3-0,5 \mathrm{~cm}$; ovário ca. $1,5 \times 0,3$ $\mathrm{cm}$.

Bahia, Espírito Santo, Maranhão, Minas Gerais, Pará, Rio de Janeiro e São Paulo (Pabst \& Dungs 1975; Toscano de Brito \& Cribb 2005; Barros et al. 2010). D6, E3, E7, E8: preferencialmente em caatinga e cerrado. Floresce de julho a fevereiro.

Material selecionado - Barreiras, abaixo da Cachoeira do Acaba-vida, $12^{\circ} 07^{\prime} 60^{\prime}$ 'S $, 45^{\circ} \mathrm{W}, 1$ nov. 1987 (fl. O'), L.P. Queiroz et

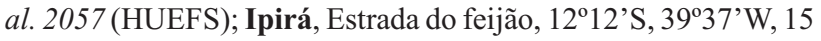
jul. 2001 (fl. O'), E.C. Smidt 187 (HUEFS); Morro do Chapéu, estrada para Bonito, $11^{\circ} 44^{\prime} 02^{\prime \prime} \mathrm{S}, 41^{\circ} 08^{\prime} 28^{\prime \prime} \mathrm{W}, 1296 \mathrm{~m}, 8$ out. 2007 (fl. @ ), C.A. Bastos 90 (HUEFS); Mucugê, estrada para

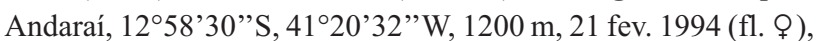
R.M. Harley et al. 14358 (SPF); Rui Barbosa, Serra do Orobó, $12^{\circ} 18^{\prime} 33^{\prime \prime}$ 'S, 40²8'57'W, 895 m, 24 fev. 2006 (fl. o'), D. Cardoso etal.1154 (HUEFS).

Catasetum hookeri parece ocupar ambientes mais úmidos do que $C$. luridum. Estas espécies são comumente confundidas, mas $C$. hookeri possui flores estaminadas não ressupinadas e labelo com lobo mediano oval, enquanto $C$. luridum apresenta flores estaminadas ressupinadas e lobo mediano arredondado. Embora não seja facilmente observável em materiais de herbário, nossas observações de campo indicam que $C$. hookeri sempre floresce no início da brotação, quando aparecem os ápices das folhas, mas o novo pseudobulbo ainda não é visível, enquanto $C$. luridum floresce após o broto já estar bem desenvolvido em um pseudobulbo.

3. Catasetum luridum (Link.) Lindl., Gen. Sp. Orch.: 156. 1832.

Figuras 1 e 2I-P.

Erva epífita. Pseudobulbo 4-20,2 × 1,1-3,3 cm, 1-7 entrenós. Folhas 18,6-56,5 × 4,3-9,6 cm, lanceoladas. Inflorescência 2-16 flores; pedúnculo 19,1-72 × 0,3-0,8 $\mathrm{cm}$; brácteas escapais $4-8,0,9-1,8 \times 0,4-0,9 \mathrm{~cm}$. Flor estaminada ressupinada; brácteas florais $2-19,0,6-1,2 \times$ $0,2-0,4 \mathrm{~cm}$; sépala dorsal $1,2-2,6 \times 0,7-1,6 \mathrm{~cm}$, ereta; sépalas laterais 1,4-2,7 ×0,9-2 cm, eretas; pétalas 1,5-2,5 $\times 0,6-1,8 \mathrm{~cm}$, sem máculas, eretas; labelo 1,6-2,7 ×0,9-2,1 $\mathrm{cm}$, envolto pelas sépalas e pétalas, carnoso, trilobado, subcalceolado, abertura oval entre os lobos, sem nervuras, lobos laterais $0,6-1,6 \times 0,4-1,3 \mathrm{~cm}$, arredondados, involutos, margem posteriormente serreada, lobo mediano 0,4-1 × 0,3-1,9 cm, levemente dobrado para frente, arredondado, sem calosidade, margem inteira a emarginada; coluna $0,8-1,2 \times 0,3-0,6 \mathrm{~cm}$, envolvida pelos lobos laterais, sépalas e pétalas; polinário $0,7-1,8 \times$ ca. 0,4 $\mathrm{cm}$; antenas 1,4-1,6 cm compr.; flor pistilada não ressupinada; sépalas suculentas, a dorsal ca. $0,9 \times 0,4 \mathrm{~cm}$, as laterais ca. $1,3 \times 0,7 \mathrm{~cm}$; pétalas ca. $1,1 \times 0,6 \mathrm{~cm}$, cartáceas a 


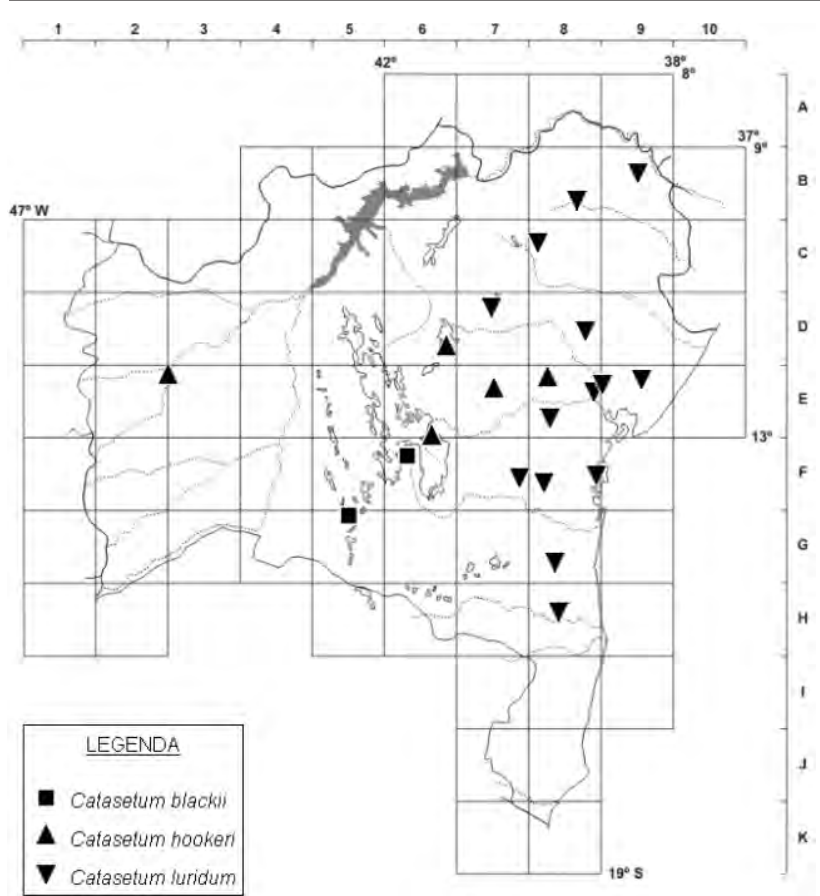

Figura 1. Mapa de distribuição de Catasetum blackii, C. hookeri e $C$. luridum na Bahia.

suculentas; labelo ca. 1,9 × 1,7 cm, calceolado, suculento; coluna ca. $0,9 \times 0,4 \mathrm{~cm}$; ovário 1,3-2,4 $\times 0,3-0,5 \mathrm{~cm}$.

Bahia, Espírito Santo e Rio de Janeiro (Pabst \& Dungs 1975; Holst 1999; Barros et al. 2010). B8, B9, C8, D7, E8, E9, F7, F8, G8: caatinga e poucos exemplares de área de mata costeira. Floresce entre fevereiro e junho, com coleta registrada também em setembro.

Material selecionado - Alagoinhas, Batalhão da Polícia Federal, $12^{\circ} 11^{\circ} 06^{\prime}$ 'S, 38 $25^{\circ} 41^{\prime \prime} \mathrm{W}, 179$ m, 24 abr. 2010 (bot.), E. Melo et al. 8091 (HUEFS); Almadina, Serra Pancadinho, 1442'20"S, 39³8'05'W, 10 mar. 1971 (fl. o'), S.P. Raimundo 1081 (CEPEC); Andorinha, Serra da Itiubá, 10¹7'35”S, 3952'19'W, 481 m, 18 fev. 2006 (fl. ơ), E.B. Souza et al. 1498 (HUEFS); Cairu, Ilha de Boipeba, 25 jun. 2000 (fr.), F. Juchum 14 (CEPEC); Camacã, estrada para Jacareci, 15²2'52"S, 39³4'11"W, 24 maio 1994 (fl. O ), W.W. Thomas et al. 10449 (CEPEC); Conceição do Coité, Serra do Mucambo, 11³0'23”'S 39¹2'10"W, 27 jun. 2010 (fl. O'), D.N. Carvalho 7 (HUEFS); Feira de Santana, Serra de São José, $12^{\circ} 15^{\prime}$ S, $38^{\circ} 58^{\prime}$ W, 12 maio 2003 (fl. Ơ ), C. van den Berg 954 (HUEFS); Glória, Terra indígena Pankararé, 09²0'S, 38²9'W, 22 abr. 2006 (fl. Ơ'), M. Colaço 110 (HUEFS); Itatim, Morro das Tocas, 12\%43'S, $39^{\circ} 42^{\prime} \mathrm{W}, 280$ m, 29 jun. 1996 (fl. ơ), E. Melo et al. 1616 (HUEFS); Jacobina, estrada para Várzea Nova, 11\%07'33"S, 40³6'2"W, 527 m, 24 set. 2004 (fl. ơ), E.L. Borba et al. 1950 (HUEFS); Maracás, EBDA, 1331'37'S, 406'52”W, 748 m, 23 abr. 2002 (fl. $0^{\prime)}$ ), R.P. Oliveira et al. 778 (HUEFS); Santa Brígida, 09²0'S, 38²9'W, 26 jun. 1982 (fl. O' ), L.P. Queiroz 394 (HUEFS); Santa Terezinha, estrada para Itatim, 12\%42'18”S, 3942'44”'W, 27 maio 1987 (fl. O ), L.P. Queiroz et al. 1528 (HUEFS); Uauá, Serra do Jerônimo, 0943'23'S, 39¹9'56”', 7 m, 30 mar. 2000 (fl. ㅇ ), L.J. Alves 13 (HUEFS); Wenceslau

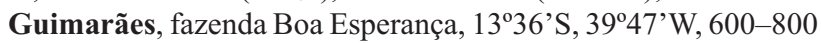
m, 15 maio 1992 (fl. o'), W.W. Thomas et al. 9357 (CEPEC).
Catasetum luridum possui morfologia floral similar a $C$. hookeri, como mencionado nos comentários de C. hookeri. É a espécie mais comum no estado, ocorrendo em áreas de caatinga e sendo coletada comumente. Cresce quase exclusivamente como epífita sobre licuri.

4. Catasetum macrocarpum Rich. ex Kunth, Syn. Pl. 1: 331.1822.

Figuras 3 e 4 A-D.

Erva epífita. Pseudobulbo 4,9-13,9 × 0,7-1,9 cm, 3-5 entrenós. Folhas 16-27 × 2,7-5,4 cm, lanceoladas. Inflorescência 2-7 flores; pedúnculo 16,5-26,9 × 0,2-0,6 $\mathrm{cm}$; brácteas escapais ca. 5, 0,9-1,2 × 0,4-0,8 cm. Flor estaminada não ressupinada; brácteas florais $2-7,0,8-1,1 \times$ ca. 0,3 cm; sépala dorsal $4-4,4 \times 1,6-1,8 \mathrm{~cm}$, patente; sépalas laterais $4,3-5 \times 2,1-2,4 \mathrm{~cm}$, patentes; pétalas 4,1-4,5 × 2-2,3 cm, com máculas castanhas, patentes; labelo 1,9-2,3 × 2-4,3 cm, semienvolto pelas sépalas e pétalas, carnoso, bilobado, campanulado, abertura oval entre os lobos, nervado, lobos laterais $0,6-1,8 \times 0,6-2,1 \mathrm{~cm}$, ovados, eretos, margem inteira, lobo mediano indefinido, calosidade central entre os lobos; coluna 3,1-4 × ca. 0,5 cm, livre; polinário ca. $1,7 \times 0,8 \mathrm{~cm}$; antenas $2,3-2,6 \mathrm{~cm}$ compr.; flor pistilada não vista.

Ocorre na Colômbia, Guiana, Suriname, Peru, Venezuela e Trinidad e Tobago. No Brasil, há registros para os estados do Amazonas, Bahia, Espírito Santo, Maranhão, Pará, Pernambuco, Rio de Janeiro, Roraima e Tocantins (Pabst \& Dungs 1975; Toscano de Brito \& Cribb 2005; Barro et al. 2010). D9, E8, E9, F8: mata ombrófila. Floresce entre fevereiro e julho.

Material selecionado - Cachoeira, estrada para Bananeiras, 12³2'S, 395'W, 40-120 m, fev. 1981 (fl. o'), s.c. (ALCB 09351); Catu, jul. 2000 (fl. ơ), R.M.O. Alves 89 (HRB); Entre Rios, fazenda Rio do Negro, $12^{\circ} 01^{\prime}$ 'S, $38^{\circ} 02^{\prime}$ 'W, 27 abr. 2010 (fl. O'), A.V. Popovkin 691 (HUEFS); Jussari, estrada para Palmira, 159'37'S, 39॰32'10”'W, 14 fev. 2004 (fl. o'), P. Fiaschi et al. 1988 (CEPEC); Muritiba, Serra da Copioba, s.d. (fl. ơ), E.C. Smidt 317 (HUEFS).

Catasetum macrocarpum foi uma das espécies que apresentou menos problemas de identificação, sendo facilmente reconhecida por suas flores estaminadas não ressupinadas, labelo carnoso com lobo mediano indefinido, calosidade central entre os lobos laterais eretos e pétalas com máculas castanhas.

5. Catasetum purum Nees \& Sinning, Pl. Hort. Bonn. Icon. $1: 1.1824$

Figuras 3 e 4E-H.

Erva epífita ou terrestre. Pseudobulbo 3,2-14,6 $\times$ 0,9-2,2 cm, 3 ou 4 entrenós. Folhas 5,4-47,5 × 0,9-4,8 cm, lanceoladas. Inflorescência 3-12 flores, pedúnculo $15,5-32 \times 0,3-0,5 \mathrm{~cm}$; brácteas escapais 3-5, 0,7-1,4× $0,3-0,6 \mathrm{~cm}$. Flor estaminada não ressupinada; brácteas florais 2-14, 0,7-1,1 × ca. 0,3 cm; sépala dorsal 2,5-3 $\times$ 0,9-1,6 cm, ereta; sépalas laterais 2,4-3 × 1,1-1,8 cm, eretas; pétalas 2,7-2,9 ×0,8-1,3 cm, sem máculas, eretas; labelo $1,2-1,7 \times 1-1,7 \mathrm{~cm}$, semienvolto pelas sépalas e pétalas, carnoso, trilobado, calceolado, com abertura pandurada, sem nervuras, lobos laterais $0,7-1,2 \times 0,3-0,8$ 


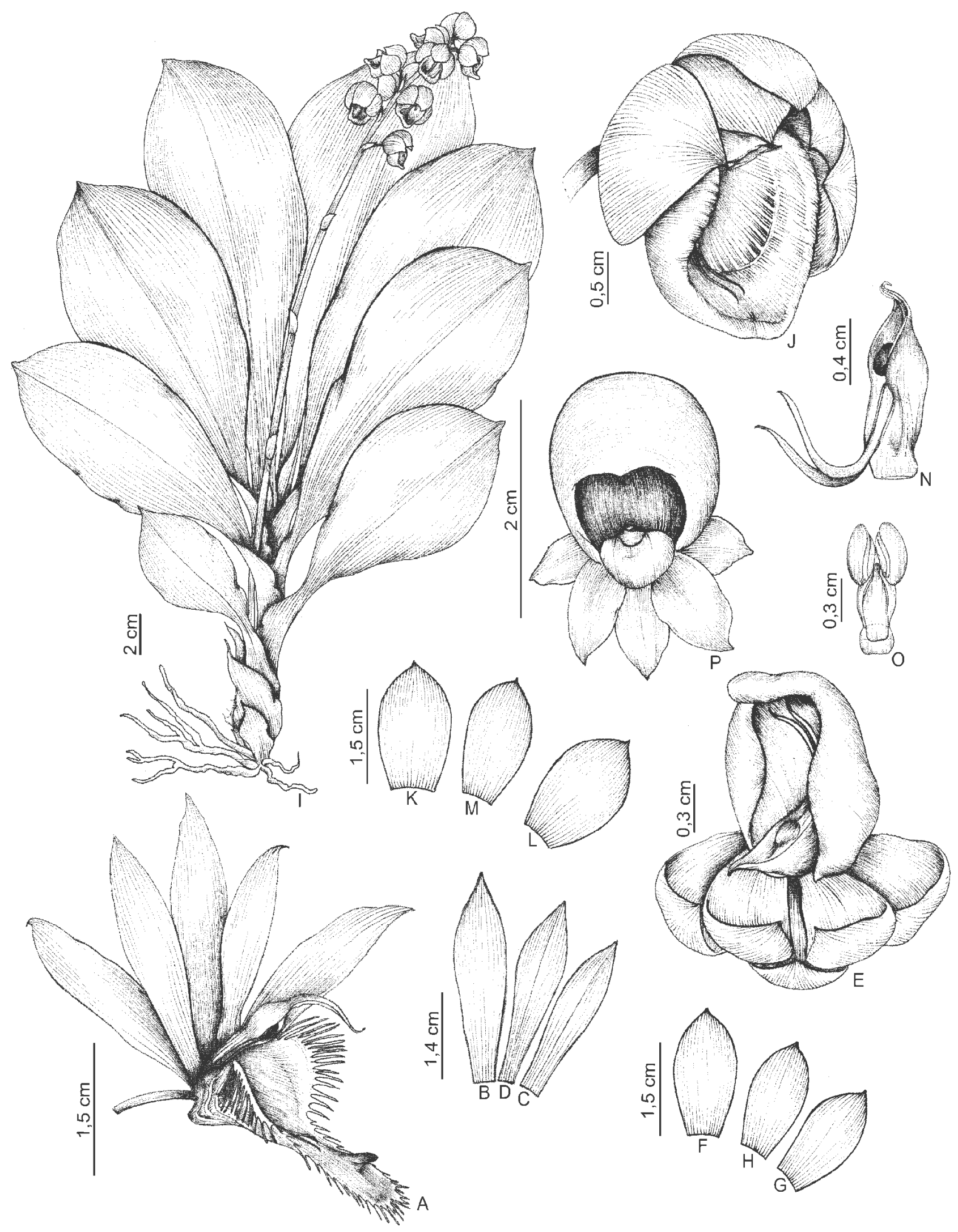

Figura 2. A-D. Catasetum blackii: A- flor ơ; B- sépala dorsal; C- sépala lateral; D- pétala (Guedes ALCB 24306). E-H. C. hookeri: E- flor ơ; F- sépala dorsal; G- sépala lateral; H- pétala (Nonato 954). I-P. C. luridum: I- hábito; J- flor Ơ; K- sépala dorsal; L- sépala lateral; M-pétala; N-coluna com antenas; O-polinário; P- flor † (I-O- Borba 1950; P- Queiroz 1528).

Sitientibus série Ciências Biológicas 12(1): 83-89. 2012. 
$\mathrm{cm}$, arredondados, involutos, margem denticulada, lobo mediano $0,4-0,6 \times 0,3-0,5 \mathrm{~cm}$, involuto, arredondado, pequena calosidade na parte interna, margem inteira; coluna 1,1-1,4× ca. $0,5 \mathrm{~cm}$, livre; polinário ca. $0,8 \times 0,2 \mathrm{~cm}$; antenas 0,6-1,7 cm compr.; flor pistilada não vista.

Bahia, Espírito Santo e São Paulo (Pabst \& Dungs 1975; Holst 1999; Barros et al. 2010). E8, E9, H8, I8: mata ombrófila. Floresce de dezembro a julho.

Material selecionado - Catu, jul. 2000 (fl. (o'), R.M.O. Alves s.n. (HRB 42468); Jussari, Serra do Teimoso, $15^{\circ} 10^{\prime} \mathrm{S}, 39^{\circ} 35^{\prime} \mathrm{W}$, 18 maio 2004 (fl. o'), C. van den Berg et al. 1407 (HUEFS); Ilhéus, estrada para Itabuna, 1446’38'S, 3905'28'W, 15 jan. 1995 (fl. ○'), W.W. Thomas et al. 10775 (CEPEC); Mucuri, 18 jun. 1985 (fl. o'), G. Hatschbach \& F.J. Zelma 49462 (MBM); Salvador, Bairro da Graça, 9 dez. 1983 (fl. ơ), L.P. Queiroz 780 (HUEFS); Santa Cruz Cabrália, Estação Ecológica do Pau Brasil, 16¹6'60"S, 3901'60"W, 21 mar. 1978 (fl. o'), S.A. Mori et al. 9779 (CEPEC); Santa Terezinha, 1246'19'S, 39³1'24”W, abr. 1998 (fl. O'), C.O. Azevedo \& R.M.O. Alves 54 (HRB).

Catasetum purum também compõe o grupo de espécies semelhantes a $C$. hookeri. É diferenciada das demais por apresentar lobos mediano e laterais involutos de margem denticulada (Hoehne 1942). Catasetum micranthum Barb.Rodr. foi aqui considerada sinônimo de C. purum. Mencionada por Holst (1999) como uma espécie enigmática da Bahia, a ilustração de $C$. micranthum de Barbosa Rodrigues em Sprunger (1996) é idêntica aos materiais de $C$. purum ocorrentes no estado. Além disso, $C$. micranthum é citada para a Bahia, Paraná e Espírito Santo (Pabst \& Dungs 1975; Barros et al. 2010), distribuição semelhante a C. purum, o que nos leva a crer que materiais de $C$. micranthum devam ser considerados como parte da variação dessa mesma espécie.

6. Catasetum roseo-album (Hook.) Lindl., Edwards's Bot. Reg. 26: 65. 1840.

Figuras 3 e 4I-O.

Erva terrestre. Pseudobulbo 6,7-20,2 × 0,7-3,5 cm, 2-9 entrenós. Folhas 9-38,3 × 0,4-7,9 cm, lanceoladas. Inflorescência 2-15 flores; pedúnculo 27,7-59 × 0,2-0,4 $\mathrm{cm}$; brácteas escapais 3-7, 0,6-1,2 × 0,3-0,9 cm. Flor estaminada não ressupinada; brácteas florais 2-17, 0,5-8 $\times$ $0,1-0,7 \mathrm{~cm}$; sépala dorsal $0,4-1,6 \times 0,1-0,5 \mathrm{~cm}$, patente; sépalas laterais $1,3-1,8 \times 0,2-0,6 \mathrm{~cm}$, patentes; pétalas 1,3-1,9 × 0,4-0,6 cm, sem máculas, patentes; labelo 1,4-2,9 $\times$ 0,8-2 cm, livre, membranoso, trilobado, cuculado, abertura oval entre os lobos, nervuras presentes ou ausentes; lobos laterais ca. $0,3 \times 1,2 \mathrm{~cm}$, forma irregular, revolutos ou eretos, margem fimbriada, lobo mediano $0,2-1,2 \times 0,2-1,6 \mathrm{~cm}$, revoluto ou ereto, triangular, sem calosidade na face adaxial, margem fimbriada; coluna 0,3-1,1 ×0,3-0,7 cm, livre; polinário 0,6-0,9 × 0,3-0,4 cm; antenas inconspícuas; flor pistilada não ressupinada; sépalas membranáceas, a dorsal ca. $1,4 \times 0,5 \mathrm{~cm}$, as laterais ca. 1,5 ×0,6 cm; pétalas ca. 1,5 ×0,4 cm, membranáceas; labelo ca. $1,7 \times 1,1 \mathrm{~cm}$, profundamente campanulado, suculento; coluna ca. $0,4 \times 0,3 \mathrm{~cm}$; ovário ca. $1,9 \times 0,3 \mathrm{~cm}$.

Ocorre desde o norte da América do Sul até o sul da Bahia (Hoehne 1942). No Brasil, já foi citada para os

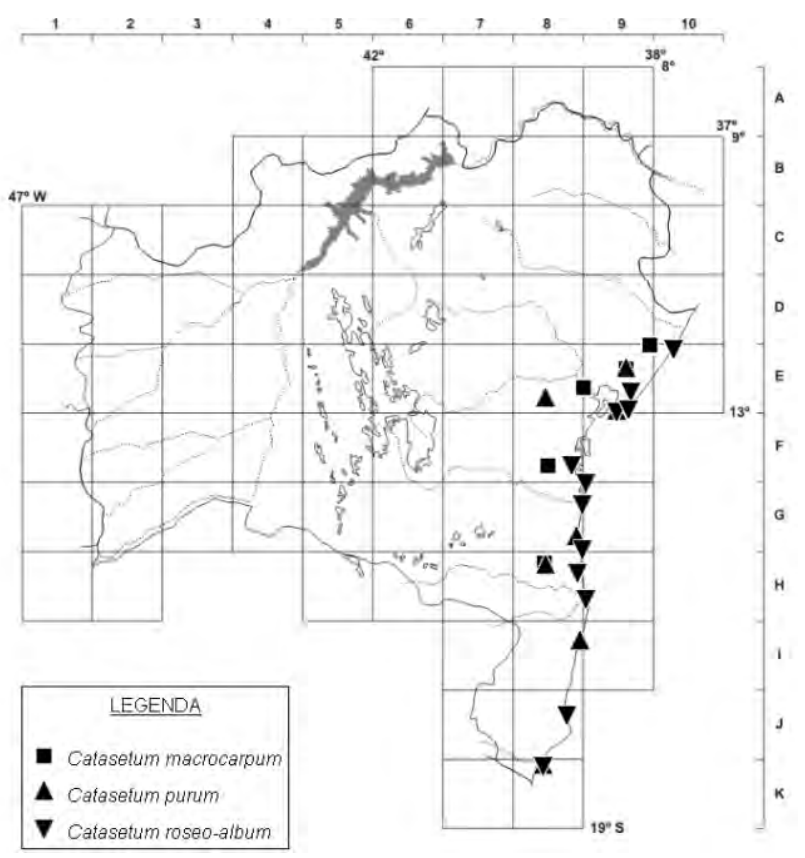

Figura 3. Mapa de distribuição de Catasetum macrocarpum, C. purum e C. roseo-album na Bahia.

estados do Amazonas, Bahia, Ceará, Espírito Santo, Maranhão, Pará, Pernambuco, Rio de Janeiro e Roraima (Pabst \& Dungs 1975; Barros et al. 2010). E9, E10, G9, K8: prioritariamente em restinga. Floresce de maio a novembro.

Material selecionado - Camaçari, $12^{\circ} 41^{\prime} \mathrm{S}, 38^{\circ} 19^{\prime} \mathrm{W}$, out. 2001 (fl. ơ), A.L. Cotias et al. s.n. (ALCB 54366); Canavieiras, estrada para Santa Luzia, 18 out. 1998 (fl. ơ), G. Hatschbach et. al. 68636 (MBM); Conde, 7 nov. 1995 (fl. ơ), M.C. Ferreira \& T. Jost 813 (CEPEC); Esplanada, Linha Verde, 1203'38'S, 3741'39”'W, 20 m, 18 ago. 2003 (fl. ᄋ), A.S. Conceição \& K.R.B. Leite 679 (HUEFS); Ilhéus, Olivença, 145' $\mathrm{S}, 39^{\circ} \mathrm{W}, 20$ out. 2005 (fl. O'), E.C. Smidt 650 (HUEFS); Itacaré, estrada para Serra Grande, 26 ago. 1992 (fr.), A.M. Amorim et al. 722 (CEPEC); Ituberá, estrada para Pratigi, 5 out. 1998 (fl. O), G. Hatschbach et al. 68556 (MBM); Maraú, BR-03 Saquaíra, 1359'18”S, 38 57'08'W, 6 set. 1999 (fl. O"), A.M. Carvalho et al. 6778 (HUEFS); Mucuri, estrada para Nova Viçosa, 10 m, 20 maio 1980 (fl. O"), L.A.M. Silva \& T.S. Santos 755 (CEPEC); Olivença, Parque dos Orixás, 16 out. 1998 (fl. ㅇ), G. Hatschbach et al. 68566 (MBM); Prado, estrada para Itamaraju, 12 ago. 1995 (fl. ণ'), G. Hatschbach \& J.T. Motta 63045 (MBM); Salvador, Dunas de Itapuã, 1256'S, 38²1'W, 9 ago. 1986 (fl. O), L.P. Queiroz 945 (HUEFS); Una, estrada para Olivença, 28 out. 1971 (fl. ơ), R.S. Pinheiro 1674 (CEPEC).

Material adicional - PERNAMBUCO: Recife, Apipucos, nov. 1837 (fl. ơ), G. Gardner 1160 (holótipo, foto HUEFS).

Catasetum roseo-album é caracterizada pela presença de fímbrias no labelo (Bicalho 1960) e antenas inconspícuas, além de ser a espécie com a menor flor dentre as que ocorrem no estado. É similar a C. discolor Lindl., espécie citada para a Bahia por outros autores (Pabst \& Dungs 1975; Holst 1999; Barros et al. 2010). No entanto, $C$. roseo-album ocorre em bancos de areia ao longo do litoral e, na Bahia, foi encontrada exclusivamente em restinga, 


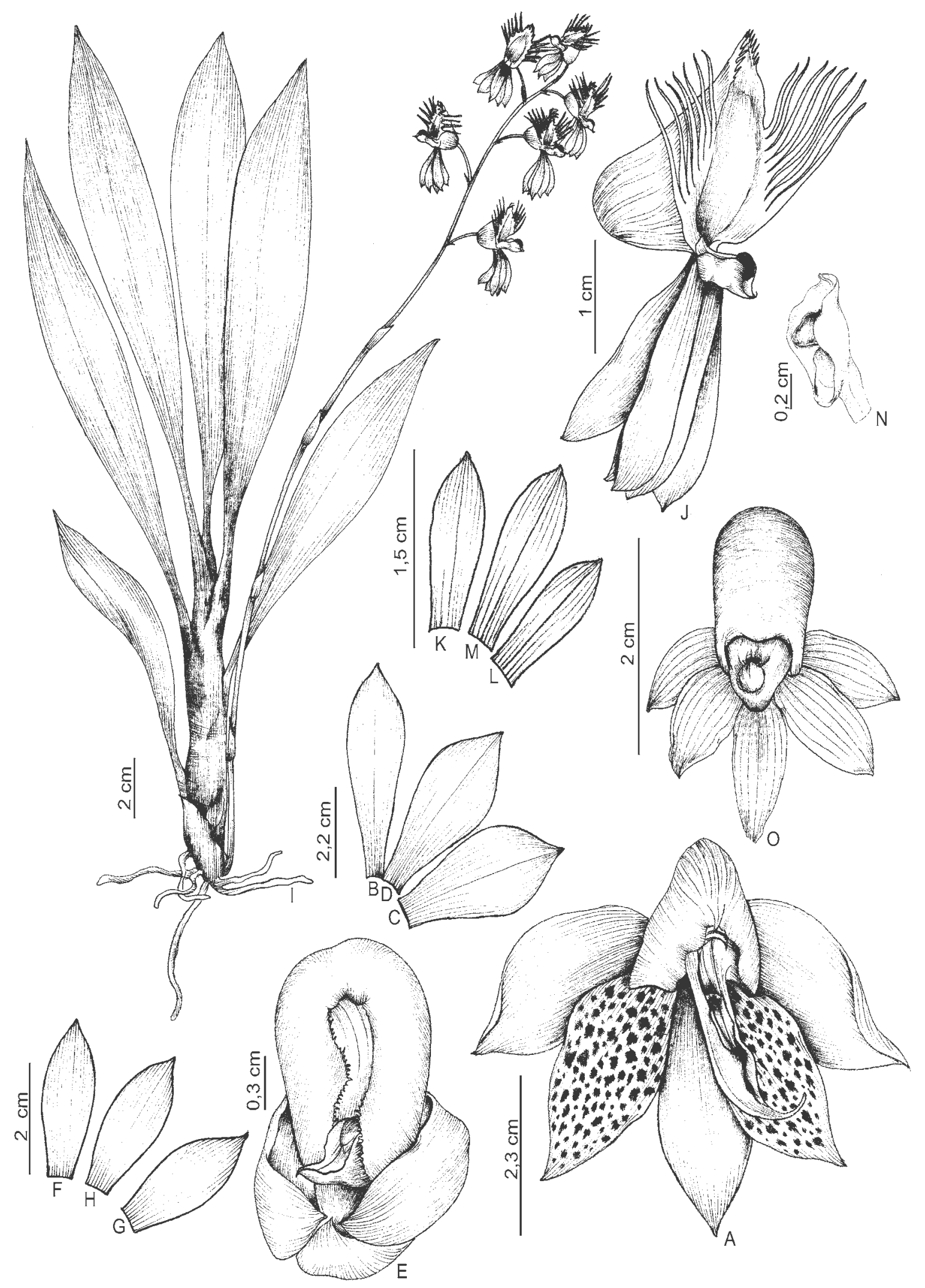

Figura 4. A-D. Catasetum macrocarpum: A- flor O’; B- sépala dorsal; C- sépala lateral; D- pétala (Alves 89). E-H. C. purum: E- flor O’; F- sépala dorsal; G- sépala lateral; H- pétala (van den Berg 1407). I-O. C. roseo-album: I- hábito; J- flor ơ; K- sépala dorsal; L- sépala lateral; M- pétala; N- coluna com parte do pedicelo; O- flor (I- Carvalho 6778 e van den Berg 1000; J-N-Nascimento 88; O- Queiroz 1401).

Sitientibus série Ciências Biológicas 12(1): 83-89. 2012. 
enquanto $C$. discolor ocorre no norte da América do Sul, ocasionalmente como rupícola ou epífita (Holst 1999). Alguns autores chegaram a considerá-la sinônimo de $C$. roseo-album (Hoehne 1942; Sprunger 1996), enquanto outros acreditam que $C$. roseo-album seja uma variedade de C. discolor, ou ainda, que se trata de um híbrido natural entre C. discolor e C. longifolium Lindl. (Holst 1999). É difícil separar essas duas espécies morfologicamente, mas as diferenças na distribuição geográfica e hábitat podem refletir alguma separação entre elas. Estudos relacionados à variabilidade genética poderão auxiliar a esclarecer este impasse taxonômico, a exemplo de Almeida (2009) na delimitação de Encyclia dichroma (Lindl.) Schltr. e E. ghillanyi Pabst.

Catasetum gardneri Schltr., citada por Holst (1999), e C. ciliatum Barb.Rodr., tratada como variedade de $C$. discolor por ele, foram aqui consideradas sinônimos em $C$. roseo-album, uma vez que ambas apresentam o mesmo padrão morfológico e ocorrem ao longo do litoral, em restinga, sendo citadas por diversos autores para os mesmos estados brasileiros.

\section{Agradecimentos}

Os autores agradecem à Fundação de Amparo a Pesquisa do Estado da Bahia (FAPESB) pela concessão da bolsa de iniciação científica à primeira autora; às pessoas vinculadas ao laboratório TAXON e ao HUEFS pela colaboração; aos herbários ALCB, HRB e CEPEC, por terem enviado os materiais necessários para o estudo e à Carla de Lima pelas ilustrações.

\section{REFERÊNCIAS}

Almeida, P.R.M. 2009. Associação Micorrízica e Estudo da Variabilidade Intra e Interespecífica em Populações de Encyclia dichroma (Lindl.) Schltr. e E. ghillanyi Pabst (Orchidaceae). Dissertação de Mestrado. Universidade Estadual de Feira de Santana.

Barros, F.; Vinhos, F.; Rodrigues, V.T.; Barberena, F.F.V.A. \& Fraga, C.N. 2010. Orchidaceae. In: R.C. Forzza, J.F.A. Baumgratz, C.E.M. Bicudo, A.A. Carvalho Jr, A. Costa, D.P. Costa, M. Hopkins, P.M. Leitman, L.G. Lohmann, L.C. Maia, G. Martinelli, M. Menezes, M.P. Morim, M.A.N. Coelho, A.L. Peixoto, J.R. Pirani, J. Prado, L.P. Queiroz, V.C. Souza, J.R. Stehmann, L.S. Sylvestre, B.M.T. Walter \& D. Zappi (eds), Catálogo de Plantas e Fungos do Brasil. Vol. 2. Instituto de Pesquisas Jardim Botânico do Rio de Janeiro, Rio de Janeiro, p. 1344-1426.

Bicalho, H.D. 1960. Contribuição à Sistemática do Gênero Catasetum L. C. Rich. (Orchidaceae). Tese de Doutorado. Escola Superior de Agricultura Luis de Queiroz.

Dressler, R.L. 1993. Phylogeny and Classification of the Orchid
Family. Dioscorides Press, Portland.

Hoehne, F.C. 1942. Orchidaceas, 103 - Catasetum. In: Flora Brasílica. Vol. 12(6). Departamento de Botânica do Estado, São Paulo, p. 58-133.

Holst, A.W. 1999. The World of Catasetums. Timber Press, Portland.

Pabst, G.F.J. \& Dungs, F. 1975. Orchidaceae Brasiliensis. Vol. 1. Brucke-Verlag, Hildesheim.

Pabst, G.F.J. \& Dungs, F. 1977. Orchidaceae Brasiliensis. Vol. 2. Brucke-Verlag, Hildesheim.

Pridgeon, A.M.; Cribb, P.J.; Chase, M.W. \& Rasmussen, F.N. 1999. Genera Orchidacearum. Vol. 1. Oxford University Press, New York.

Sprunger, S. 1996. Catalogue. In: S. Sprunger, P. Cribb \& A.L.V. Toscano de Brito (eds), Iconographie des Orchidées du Brésil. Vol. 1. Friedrich Reinhardt Verlag, Basle, p. 344-519.

Toscano de Brito, A.L.V. \& Cribb, P. 2005. Orquídeas da Chapada Diamantina. Nova Fronteira, São Paulo.

\section{Lista de Exsicatas}

Alves, L.J. 13 (3); Alves, R.M.O. 89 (4), s.n. HRB 42468 (5); Amorim, A.M. 722 (6), 723 (6); Azevedo, C.O. 54 (5); Bastos, C.A. 90 (2); Belém, R.P. 3543 (6); Borba, E.L. 1950 (3); Cardoso, D. 1041 (6), 1154 (2); Carvalho, A.M. 6778 (6); Carvalho, D.N. 7 (3); Chautems, A. 6 (6); Colaço, M. 110 (3); Conceição, A.S. 679 (6); Cotias, A.L. s.n. ALCB 54366 (6); Ferreira, M.C. 813 (6); Fiaschi, P. 1988 (4); Guedes, M.L. s.n. ALCB 024306 (1); Harley, R.M. 14358 (2), 22102 (6); Hatschbach, G. 49462 (5), 50730, 63045, 68556, 68566, 68636, 68895 (6); Juchum, F. 14 (3); Kuhn Neto, R. 13 (6); Lucca, C.F. 21 (3); Melo, E. 1498 (3), 1616 (3); 8091 (3); Mori, S.A. 9779 (5), 11988, 12789 (6); Muller, H. s.n. SP 18606 (6), s.n. SP 221800 (6);
Nascimento, C.B. 88 (6); Noblick, L.R. 2748 (3), 4456 (6); Nonato, F.R. 953, 954 (2); Oliveira, C.M. 6 (6); Oliveira, R.P. 778 (3); Pinheiro, R.S. 1674 (6), 1811 (5); Pinto, G.C.P. 183/84, s.n. CEPEC 40504 (3); Popovkin, A.V. 691, 846 (4); Queiroz, L.P. 394, 569, 581 (3), 780 (5), 934, 945, 1401, 1403 (6), 1528 (3), 2057 (2); Raimundo, S.P. 1081 (3); Santos, T.S. 2265 (5), 3527 (6); Silva, L.A.M. 755, 3800 (6); Smidt, E.C. 187 (2), 317 (4), 650, 680 (6); Souza, E.B. 1498 (3); Thomas, W.W. 9357, 10449 (3), 10775 (5); Toscano de Brito, A.L.V. 1824 (1); van den Berg, C. 954 (3), 1000 (6), 1407 (5); Vinha, S.G. 58 (6); s.c. ALCB 09351 (4), SP 31176 (5), SP 65490 (6). 